\title{
Achieving Optimal Degrees of Freedom for an Interference Network with General Message Demand
}

\author{
Zainalabedin Samadi \\ School of Electrical Engineering \\ Iran University of Science \& \\ Technology \\ Tehran, Iran \\ z.samadi@gmail.com
}

\author{
Vahid Tabataba Vakili \\ School of Electrical Engineering \\ Iran University of Science \& \\ Technology \\ Tehran, Iran \\ vakily@iust.ac.ir
}

\author{
Farzan Haddadi \\ School of Electrical Engineering \\ Iran University of Science \& \\ Technology \\ Tehran, Iran \\ haddadi@iust.ac.ir
}

\begin{abstract}
The concept of degrees of freedom (DoF) has been adopted to resolve the difficulty of studying the multi-user wireless network capacity regions. Interference alignment (IA) is an important technique developed recently for quantifying the DoF of such networks. In the present study, a single-hop interference network with $K$ transmitters and $N$ receivers is taken into account. Each transmitter emits an independent message and each receiver requests an arbitrary subset of the messages. Using the linear IA techniques, the optimal DoF assignment has been analyzed. Assuming generic channel coefficients, it has been shown that the perfect IA cannot be achieved for a broad class of interference networks. Analytical evaluation of DoF feasibility for general interference channels (IFCs) is complicated and not available yet. Iterative algorithm designed to minimize the leakage interference at each receiver is extended to work with general IFCs. This algorithm provides numerical insights into the feasibility of IA, which is not yet available in theory.
\end{abstract}

Keywords-interference channel; interference alignment; degrees of freedom; channel state information

\section{INTRODUCTION}

Despite the intensive research on multi-user wireless communication networks over the past three decades, the subject is still not well understood from information-theoretic perspective. Capacity limits of many multi-user networks are still unknown (even for a small number of users). For example, the capacity region of a two-user single-antenna interference channel (IFC) is still unknown, though the region can be bounded up to a constant value [1]. Researchers have derived various approximations of the capacity region of the IFCs. For example, the maximum total degrees of freedom (DoF) correspond to the first order approximation of the sum-rate capacity in high SNR regime. DoF is not a new concept, it is widely known as the multiplexing gain in point-to-point communication scenarios. It was termed as spatial DoF in [2], referring to the maximum multiplexing gain. In the present study, the maximum multiplexing gain is presumed to be the total DoF. The study of DoF of interference networks was pioneered in [3]. In [2], the total DoF of two-user multipleinput multiple-output IFC is provided. Interference alignment (IA) is a powerful scheme developed recently for quantifying the DoF region of multi-user networks. IA refers to the construction of signals in such a way that their mutual interference aligns at the receivers, facilitating simple interference cancellation techniques. The remaining dimensions are designated for communicating the desired signal, keeping it free from interference. IA was first introduced in [4], and clarified in [5]. The analysis of DoF is shown to be very useful in revealing the capacity potential of the IFCs. The channel capacity of IFC is limited with large number of users. Authors in $[3,6]$, showed that the total number of $\eta=K M / 2$ DoF can be achieved asymptotically via infinite time (frequency) expansion under block fading channel for a K-user $\mathrm{M}$ antenna IFC. This result indicates that the capacity of each user is unbounded regardless of the user number $\mathrm{K}$. The achieving method is based on IA.

The principal assumption enabling this surprising result is that the channel extensions are exponentially long in $\mathrm{K}$ and are generic (e.g., drawn from a continuous probability distribution). However, there is an important distinction between perfect IA schemes and partial IA schemes. Perfect IA schemes are able to exactly achieve the outer bound of the DoF with a finite symbol extension of the channel. In contrast, partial IA schemes pay a penalty in the form of the overflow room required to "almost" align interference. Study of the design and feasibility of linear beamforming for IA without symbol extensions has received significant attention [7-10].

In general, linear IA can be described by a set of bilinear equations which correspond to the zero-forcing conditions at each receiver. One can count the number of "independent unknowns" and the number of scalar equations in this quadratic system defining IA to verify if the total number of variables exceeds the total number of constraints in the system of equations. If a system has more variables than constraints, it is called a proper system. Otherwise, it is called an improper system [7]. While it is known that almost all improper systems are infeasible [8-9], the feasibility of the proper systems is still an area of active research. In [8-10], a set of sufficient conditions for feasibility are established. In this paper, we consider the case of interference networks with general message demands. In this setup, there are $\mathrm{K}$ transmitters and $\mathrm{N}$ receivers, each equipped with $\mathrm{M}$ antennas. Each transmitter 
emits a unique message and each receiver is interested in an arbitrary subset of the messages. This construction is a generalization of the multiple unicasts scenario considered in [6], to the case where each receiver is interested in an arbitrary subset of the transmitted messages. DoF region for this network is evaluated in [11]. In this work, we evaluate how to optimally allocate DoFs to the transmitters of this architecture to achieve the total DoF of such a network.

As an alternative to the closed form designs, several algorithmic approaches have been proposed in the literature. Many of those methods aim to minimize the interference leakage at each receiver so that -at the best case-the IA can perfectly be attained. The suggested insight for their effectiveness is that when perfect IA is possible, the interference leakage will be zero and such algorithms may obtain the optimal solutions. In this paper, we are mostly concerned with the feasibility of the perfect IA. We have extended the iterative IA algorithm developed in [12], to the case of general IFC to evaluate IA feasibility of different scenarios.

The rest of the paper is organized as follows. The system model is introduced in Section II. In Section III, it is argued why linear IA scheme, over a single antenna IFC, cannot achieve the total number of DoF with a limited number of channel extensions. In Section IV, an iterative IA algorithm is proposed to achieve optimal DoF of an IFC with general message demands. Simulation results are provided in Section $\mathrm{V}$, and concluding remarks are presented in Section VI.

\section{SYSTEM MODEL}

Consider a $\mathrm{K} \times \mathrm{N}$ user single-hop interference network with $\mathrm{K}$ transmitters and $\mathrm{N}$ receivers. We assume that all the transmitters and receivers have $\mathrm{M}$ antennas. An illustration of the system model is shown in Figure 1. Each transmitter has one and only one independent message. Each receiver can request an arbitrary set of messages from multiple transmitters. Let $S_{j}, j \in[\mathbf{N}]$ be the set of indices of those transmitted messages requested by receiver $j$, where $[\mathbf{N}]$ is defined as $[\mathbf{N}]=\{1, \ldots, \mathbf{N}\}$ and $\bar{S}_{j}$ is the set of indices of those transmitted messages contributing to the interference at receiver $j$. Obviously, $S=S_{j} \cup \bar{S}_{j}$ is the set of all active transmitters, where active transmitters are defined as the transmitters with assigned DoF greater than zero. All transmitters share a common bandwidth and want to achieve the maximum possible sum rate along with a reliable communication. Channel output at receiver $j$ and over time slot, $t \in \mathbb{N}$ is characterized by the following input-output relationship:

$$
\begin{aligned}
Y^{[j]}(t) & =H^{[j 1]}(t) X^{[1]}(t)+H^{[j 2]}(t) X^{[2]}(t) \cdots \\
& +H^{[j K]}(t) X^{[K]}(t)+Z^{[j]}(t),
\end{aligned}
$$

where $1 \leq j \leq N$ is the user index, $X^{[k]}(t)$ is the $M \times 1$ transmitted signal of transmitter $k, H^{[j k]}(t), 1 \leq k \leq K$ is the $M \times M$ matrix of fading factors of the channel from transmitter $k$ to receiver $j$. The channel fading factors at different time instants are assumed to be independently drawn from a continuous distribution. $Z^{[j]}(t)$ is the additive white Gaussian noise at receiver $j$. The noise terms are all assumed to be drawn independently from a Gaussian independent identically distribution with zero mean and unit variance. It is also assumed that all transmitters are subject to a power constraint $P$ :

$$
\mathrm{E}\left(\left\|X_{k}(t)\right\|^{2}\right) \leq P, \quad k \in[\mathbf{K}]
$$

where $\mathrm{E}$ is the expectation taken over time. Hereafter, time index is omitted for convenience. Let $\tau$ denote the duration of the time expansion in number of symbols. From this point and below, we use uppercase bold fonts to denote the timeexpanded signals.

$\mathbf{H}^{[\mathrm{jk}]}=\operatorname{diag}\left(H^{[j k]}(1), H^{[j k]}(2),, \ldots H^{[j k]}(\tau)\right)$, is a $M \tau \times M \tau$ block diagonal matrix. Denoting the beamforming matrix of the transmitted message $k$ as $\mathbf{V}^{[k]}$, the extended channel model can be described as follows,

$$
\begin{aligned}
\mathbf{Y}^{[j]} & =\mathbf{H}^{[j 1]} \mathbf{V}^{[1]} \mathbf{X}^{[1]}+\mathbf{H}^{[j 2]} \mathbf{V}^{[2]} \mathbf{X}^{[2]} \ldots \\
& +\mathbf{H}^{[j K]} \mathbf{V}^{[K]} \mathbf{X}^{[K]}+\mathbf{Z}^{[j]}
\end{aligned}
$$

where $\mathbf{Y}^{[j]}=\left[Y^{[j]}(1)^{T}, Y^{[j]}(2)^{T}, \ldots, Y^{[j]}(\tau)^{T}\right]^{T}$ is the $M \tau \times 1$ received signal vector over the extended channel, $\mathbf{V}^{[\mathrm{k}]}$ is the $M \tau \times d^{[k]}$ beamforming matrix of transmitter $k$, and $d^{[k]}$ is the number of independent signal symbols transmitted from transmitter $k . \mathbf{X}^{[\mathrm{k}]}$ is a $d^{[k]} \times 1$ vector of the transmitted symbols and $\mathbf{7}^{[j]}$ is a $M \tau \times 1$ received noise vector at receiver $j$. Perfect channel state information (CSI) is assumed to be available at receivers and global CSI at transmitters.

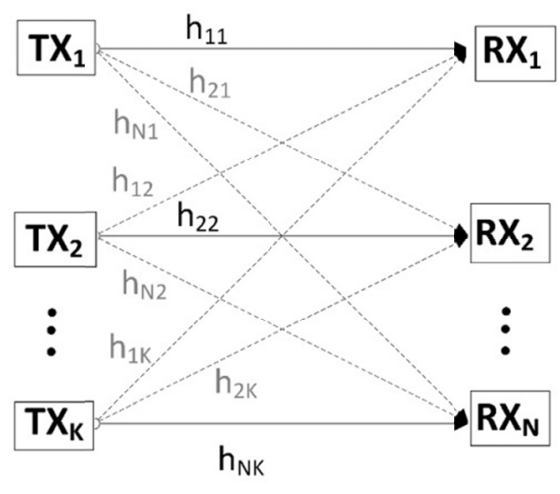

Fig. 1. $\quad K \times N$ user IFC model.

Authors in [11], have referred to the aforementioned setup as an interference network with general message demands and have derived DoF region of this setup. Denote the capacity region of such a system with power constraint $P$ as $\mathcal{C}(P)$, corresponding DoF region is defined as

$$
\begin{aligned}
\mathcal{D}= & \left\{\mathbf{d}=\left(d^{[1]}, d^{[2]}, \ldots, d^{[K]}\right) \in \mathbb{R}_{+}^{K}:\right. \\
& \exists\left(R_{1}(P), R_{2}(P), \ldots, R_{K}(P)\right) \in \mathcal{C}(P), \\
& \text { such that } \left.d^{[k]}=\lim _{P \rightarrow \infty} \frac{R_{k}(P)}{\log (P)}, k \in[\mathbf{K}]\right\} .
\end{aligned}
$$

The total DoF number is defined as:

$$
D_{s}=\max \sum_{1}^{K} d^{[k]},\left\{d^{[1]}, d^{[2]}, \ldots, d^{[K]}\right\} \in \mathcal{D} \text {. }
$$




\section{LINEAR VECTOR IA LIMITATION}

DoF region for the setup described in Section II has been derived by [11], as follows,

$$
\begin{aligned}
& \mathcal{D}= \\
& \left\{\mathbf{d} \in \mathbb{R}_{+}^{K}: \sum_{k \in S_{j}} d^{[k]}+\max _{k \in \bar{S}_{j}}\left(d^{[k]}\right) \leq M, \forall j \in[\mathbf{N}]\right\},
\end{aligned}
$$

where $\mathcal{S}_{j}$ is the set of message indices requested by receiver $j, j \in[\mathbf{N}]$. Optimal DoF assignment in an IFC with general message demands is obtained by solving the following linear programming problem;

$$
\begin{aligned}
& \mathbf{d}^{*}=\arg \max \sum_{\mathrm{k}=1}^{\mathrm{K}} d^{[\mathrm{k}]}, \\
& \text { s.t } \quad \mathbf{z} \preceq M, \\
& \mathbf{d} \succ 0 .
\end{aligned}
$$

where $\mathbf{z}$ is defined as a $G \times 1$ vector consisted of scalar elements $z_{i}=\sum_{k \in S_{i}} d^{[k]}+\max _{k \in \bar{S}_{i}} d^{[k] .} \mathbf{z} \prec M$ implies that all elements of $\mathbf{z}$ should be smaller than $M$. The solution to the feasibility problem is not known in general. In other words, given a set of randomly generated channel matrices and a degree-of-freedom allocation $\left[d^{[1]}, d^{[2]}, \ldots, d^{[K]}\right]$, it is not known if one can almost surely find the transmit and receive filters that will satisfy the feasibility conditions. If we look at the problem of finding the precoding and zero-forcing matrices when the channel coefficients are arbitrary and given, the problem is computationally intractable. In particular, [8] established that for an arbitrary $K$-user MIMO IFC, checking the achievability of a certain DoF tuple $\left[d^{[1]}, d^{[2]}, \ldots, d^{[K]}\right]$ is NP-hard when each user and transmitter has more than 2 antennas. The notion of regular IFC will be introduced below and it will be shown that perfect IA is not feasible for single antenna regular IFC. The distributed IA developed in this paper will be useful in numerically evaluating the feasibility problem for general IFC. Regular networks are defined as IFCs for which the only optimal DoF assignment is equal DoF assignment for all active transmitters. Theorem 1 implies that an IFC in which all prime receivers request the same number of transmitted messages and each transmitter sends message to an equal number of prime receivers is a regular network.

- Theorem 1. The only DoF point that achieves total number of DoF of an IFC where all receivers request the same number of transmitted messages and each transmitter sends message to equal number of receivers is

$$
\mathbf{d}=\left(\frac{M}{\beta+1}, \frac{M}{\beta+1}, \ldots, \frac{M}{\beta+1}\right)
$$

Proof. Maximum total number of DoF for this network is obtained to be $\frac{K M}{\beta+1}$, where $\beta$ is the number of requested messages for each prime receiver [11]. Obviously, total DoF is achieved by $d^{[k]}=\frac{M}{\beta+1}$. It is now intended to show that this is the only DoF point that achieves total number of DoF. It is worth noting that as prime receivers are addressed those receivers whose requested message sets are not a subset of any other requested message set. If Theorem 1 is not true, there is at least one $d^{[k]}, k \in[\mathbf{K}]$ which is strictly greater than $\frac{M}{\beta+1}$. We would also have the following lemma:

In the specified channel structure, we should have

$$
\max _{k \in \bar{S}_{j}}\left(d^{[k]}\right) \geq \frac{M}{\beta+1}, \quad \forall j \in[\mathbf{G}],
$$

where $G$ is the number of prime receivers.

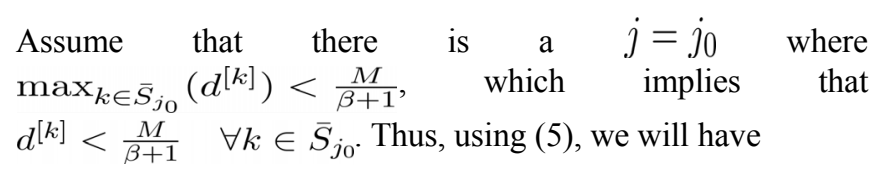

$$
\begin{aligned}
\sum_{k \in[\mathbf{K}]} d^{[k]} & \stackrel{a}{=} \sum_{k \in S_{j_{0}}} d^{[k]}+\sum_{k \in \bar{S}_{j_{0}}} d^{[k]} \\
& \leq M-\max _{k \in \bar{S}_{j_{0}}}\left(d^{[k]}\right)+\sum_{k \in \bar{S}_{j_{0}}} d^{[k]} \\
& <M+\frac{M(K-1-\beta)}{1+\beta}=\frac{M K}{1+\beta} \\
& \Rightarrow d_{\text {sum }}<\frac{K}{1+\beta} .
\end{aligned}
$$

The first equality, $a$, is derived from the fact that $[\mathbf{K}]=S_{j_{0}} \cup \bar{S}_{j_{0}}, b$ is derived from DoF region inequalities in (5) and $c$ is implied from the assumption that $\left(d^{[k]}\right)<\frac{1}{\beta+1}, \quad k \in \bar{S}_{j_{0}}$. Equation (9) contradicts the assumption that this DoF assignment achieves total DoF number. Therefore, the lemma is valid.

Based on (5), in order to characterize DoF region, we should consider $G$ inequalities of the form

$$
\sum_{k \in S_{j}} d^{[k]}+\max _{k \in \bar{S}_{j}}\left(d^{[k]}\right) \leq M, \quad \forall j \in[\mathbf{G}] .
$$

Since each message is requested by $G \beta / K$ receivers, summing all $G$ inequalities, we have

$$
G \beta / K \sum_{k \in[\mathbf{K}]} d^{[k]}+\sum_{j \in[\mathbf{G}]} d_{\max }^{j} \leq G M
$$

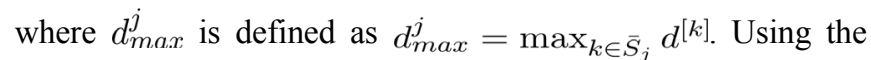
fact that there is at least one $d_{\text {max }}^{j}$ strictly greater than $\frac{M}{\beta+1}$, along with lemma 1 , it is obtained that

$$
\frac{M G}{\beta+1}<\sum_{j \in[\mathbf{G}]} d_{\max }^{j}
$$

substituting (12) in (11), we will have the following inequality: 


$$
\begin{gathered}
G \beta / K \sum_{k \in[\mathbf{K}]} d^{[k]}+\frac{G M}{\beta+1}<G M \\
\Rightarrow \sum_{k \in[\mathbf{K}]} d^{[k]}<\frac{M K}{\beta+1},
\end{gathered}
$$

which contradicts the assumption that this DoF assignment achieves total DoF number. Therefore, $d^{[k]}=\frac{M}{\beta+1}$ is the only DoF point that achieves the total number of DoF of this network.

- Theorem 2. Assuming that the channel coefficients are generic, the total number of the DoF of a single antenna regular interference network cannot be achieved using a finite extension of the channel.

Proof. It should be noted that the regular IFC should have more than $1 \mathrm{DoF}$, otherwise, the network is equivalent to a broadcast or multiple access channel. Optimal DoF assignment is not unique in this case. In fact, every $K \times 1$ vector $\mathrm{d} \succeq 0$ that satisfies $\sum_{k=1}^{K} d^{[k]}=1$ achieves optimal DoF of this network. For example, if a set, say $S_{i}$, would have $K$ or $K-1$ elements, receiver $i$ and its corresponding transmitters can be considered as a multiple access channel without losing any DoF gain, and therefore the total number of DoF of this network is 1 . There is no need for IA in this case and simple methods like time division based multiple access techniques can achieve the total number of Dof of this structure. Consider a special case of $6 \times 3$ user IFC with generalized message set, the channel structure along with requested set of messages at each receiver is shown in Figure 2.

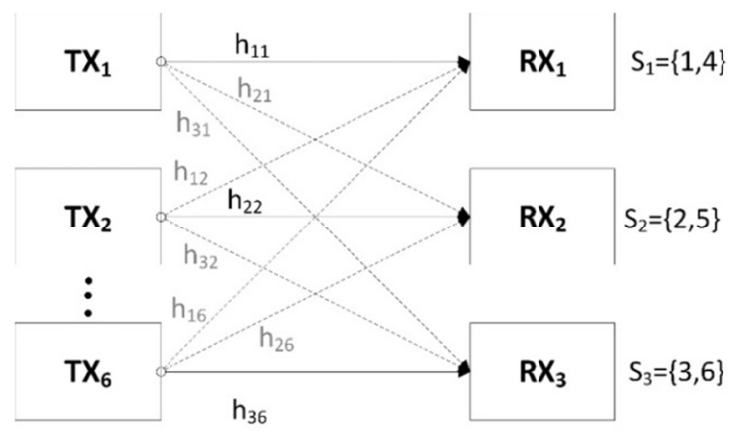

Fig. 2. $6 \times 3$ user IFC with general message demands.

The proof for the general case is similar. It is intended to achieve the outer bound of $6 / 3 \mathrm{DoF}$ for this setup. Consider 3 extensions of this channel. Over this extended channel, consider a hypothetical achievable scheme where each of the 6 messages achieves $1 \mathrm{DoF}$ if possible, using beamforming at every transmitter and zero-forcing at every receiver. Note that this is the only DoF point in achievable region that achieves total number of DoF of this network, according to Theorem 1.

Let message $W^{[k]}$ be beamformed along $3 \times 1$ vector $\mathbf{V}^{[k]}$ at transmitter $k$. Receiver $j$ intends to decode $W^{[k]} k \in S_{j}$ using zero-forcing. At receiver $j$, to decode 2 independent messages $W^{[k]}, k \in S_{i}$, the vectors corresponding to the desired messages should occupy 2 linearly independent directions. Since signals come from a space of 3 dimensions, the 4 interfering vectors must occupy the remaining 1 dimension. IA requirements can be written as follows.

$\operatorname{span}\left(\mathbf{H}^{[j k]} \mathbf{V}^{[k]}\right)=\operatorname{span}\left(\mathbf{H}^{[j m]} \mathbf{V}^{[m]}\right), \quad k, m \in \bar{S}_{j}(7)$

Thus, the total dimension of the interference is 1 and receiver $j$ can decode all its desired messages. Along with the above criteria, the desired signal vectors are required to be linearly independent of the interference dimension at each receiver. This requirement implies that,

$$
\mathrm{D}\left(\operatorname{span}\left[\mathbf{U}\left(S_{j}\right), \mathbf{U}\left(\overline{\mathbf{S}}_{\mathbf{j}}\right)\right)\right]=3,
$$

Where $\mathrm{D}(\mathrm{S})$ is defined as the dimension of a subspace $\mathrm{S}$, $\mathbf{U}\left(S_{j}\right)$ and $\mathbf{U}\left(\bar{S}_{j}\right)$ are the sets of the received signal vectors corresponding to the desired and undesired signal vectors, respectively, and 3 is the total subspace dimension available at the receivers. For example, at user $1, \mathbf{U}\left(S_{j}\right)$ is obtained as $\left[\mathbf{H}^{[12]} \mathbf{V}^{[1]}, \mathbf{H}^{[14]} \mathbf{V}^{[4]}\right.$.

Observe that (14) is a set of bilinear equations in the unknown precoding and zero-forcing filters. A feasibility question is raised as to whether a system admits perfect IA or not. Authors in [7] argued that when the channel coefficients are selected randomly and independently, the proper systems should have almost surely a perfect alignment solution. A set of bilinear equations is called proper, in other words, perfect IA is expected to be feasible almost surely when the number of variables is less than or equal to the number of equations. Moreover, [8] proved that improper system of equations is infeasible when each transmitter uses only one beamforming vector. It can be observed that the set of IA requirements, (14), constitutes an improper set of equations. The number of independent variables in this system can be obtained as

$N_{v}=\sum_{k=1}^{K}\left(L M-d^{[k]}\right) d^{[k]}+\sum_{j=1}^{N}\left(L M-n^{[j]}\right) n^{[j]}=18$

where $L$ is the number of channel extensions. $n^{[j]}=\sum_{k \in S_{j}} d^{[k \text { is }}$ the number desired streams at receiver $j$. $N_{v}$ is less than the number of equations, $N_{e}$, which is obtained as

$$
N_{e}=\sum_{i=1}^{N} n^{[j]} \bar{n}^{[j]}=24
$$

where $\bar{n}^{[j]}=\sum_{k \in \bar{S}_{j}} d^{[k}$. is the number of interfering streams at receiver $j$. Therefore, using 3 extensions of the channel or 3 antennas at each node, we cannot achieve $6 \mathrm{DoF}$ for this network. In the following, it is proved that this system of IA requirements is still infeasible using every finite extension of the channel.

Consider a $3 n$ symbol extension of the channel over which, according to Theorem 1, the only achievable scheme is the case where each of the 6 transmitters achieves $n$ DoF if possible, using beamforming at every transmitter and zero-forcing at every receiver. The $3 n \times n$ vectors $\mathbf{V}^{[j]}, j=1, \ldots, 6$ should 
satisfy IA conditions along with the linear independence condition. IA requirements at 3 receivers can be summarized as follows,

$$
\begin{gathered}
\operatorname{span}\left(\mathbf{H}^{[j k]} \mathbf{V}^{[k]}\right)=\operatorname{span}\left(\mathbf{H}^{[j m]} \mathbf{V}^{[m]}\right), \\
\forall k, m \in \bar{S}_{j} .
\end{gathered}
$$

Since diagonal channel matrices $\mathbf{H}^{[j k]}$ are almost surely full rank, after some algebraic manipulations, (18) becomes

$$
\begin{aligned}
& \operatorname{span}\left(\mathbf{T}_{k, u}^{[j]} \mathbf{V}^{[u]}\right)=\operatorname{span}\left(\mathbf{V}^{[u]}\right), \\
& j=2,3 \quad \forall u \in S_{1} \cap \bar{S}_{j}, \quad \forall k \in \bar{S}_{1} \cap \bar{S}_{j},
\end{aligned}
$$

where $\mathbf{T}_{k, u}^{[j]}$ matrices are obtained as follows,

$$
\begin{aligned}
& \mathbf{T}_{6,1}^{[2]}=\mathbf{T}_{6,4}^{[2]}=\mathbf{H}^{[26]}\left(\mathbf{H}^{[16]}\right)^{-1} \mathbf{C}_{M} \\
& \mathbf{T}_{2,1}^{[3]}=\left(\mathbf{H}^{[31]}\right)^{-1} \mathbf{H}^{[32]}\left(\mathbf{H}^{[12]}\right)^{-1} \mathbf{C}_{M} \mathbf{H}^{[21]} \\
& \mathbf{T}_{2,4}^{[3]}=\left(\mathbf{H}^{[34]}\right)^{-1} \mathbf{H}^{[32]}\left(\mathbf{H}^{[12]}\right)^{-1} \mathbf{C}_{M} \mathbf{H}^{[24]} \\
& \mathbf{T}_{5,1}^{[3]}=\left(\mathbf{H}^{[31]}\right)^{-1} \mathbf{H}^{[35]}\left(\mathbf{H}^{[15]}\right)^{-1} \mathbf{C}_{M} \mathbf{H}^{[21]} \\
& \mathbf{T}_{5,4}^{[3]}=\left(\mathbf{H}^{[34]}\right)^{-1} \mathbf{H}^{[35]}\left(\mathbf{H}^{[15]}\right)^{-1} \mathbf{C}_{M} \mathbf{H}^{[24]}, \\
& \text { and } \mathbf{C}_{\mathbf{M}} \text { is defined as } \\
& \mathbf{C}_{M}=\mathbf{H}^{[13]}\left(\mathbf{H}^{[23]}\right)^{-1} .
\end{aligned}
$$

Assuming $\mathbf{V}^{[1]}$ is of rank $n$, [13] has shown that (20) implies $n$ eigenvectors of $\mathbf{T}_{k, u}^{[j]}$ lie in $\operatorname{span}\left(\mathbf{V}^{[u]}\right), u \in S_{1}$. Since all channel matrices are diagonal, the set of eigenvectors of channel matrices, their inverse and product are column vectors of the identity matrix. Define $\mathbf{e}_{i}=\left[\begin{array}{llllll}0 & 0 & \cdots & \cdots & \cdots & 0\end{array}\right]^{T}$ and note that $\mathbf{e}_{\mathbf{i}}$ exists in $\operatorname{span}\left(\mathbf{V}^{[u]}\right), \forall u \in S_{1}$, therefore, the set of equations in (19) implies that

$$
\begin{aligned}
& \mathbf{e}_{i} \in \operatorname{span}\left(\mathbf{H}^{[j k]} \mathbf{V}^{[k]}\right), \\
& \forall(j, k) \in\{1,2,3\} \times\{1, \ldots, 6\}
\end{aligned}
$$

Thus, at receiver 1 , the desired signal $\left[\mathbf{H}^{[11]} \mathbf{V}^{[1]}, \mathbf{H}^{[14]} \mathbf{V}^{[4]}\right]$ is not linearly independent of the interference signal, $\mathbf{H}^{[12]} \mathbf{V}^{[2]}$, and hence, receiver 1 cannot fully decode $W^{[1]}$ and $W^{[4]}$ solely by zero-forcing the interference signal. Therefore, if the channel coefficients are completely random and generic, we cannot obtain $6 / 3 \mathrm{DoF}$ for the $6 \times 3$ user single antenna IFC through linear IA schemes.

Regular IFCs are mostly encountered in networks with few users. We have evaluated optimal DoF assignment for $10^{5}$ random configurations of a $10 \times 10$ IFC. Roughly, $25 \%$ of the evaluated networks were regular IFCs. It is implied that there are many cases where perfect IA for a single antenna IFC is not feasible.

\section{DISTRIBUTED IA ALGORITHM FOR GENERAL IFC}

In general, the optimal DoF solution for each specific configuration can be obtained by solving the linear programming problem (6), using methods like simplex algorithm. The closed form solution for each arbitrary requested message set structure is not straightforward. Moreover, analytical evaluation of DoF feasibility is not straightforward. Interference can be aligned in networks with single antenna nodes through the channel extension in frequency or time as long as the channel is varying across frequency or time. However, this approach requires long symbol extensions. For example, the achievable scheme presented in [11] uses at least 1536 extensions of the single $6 \times 3$ antenna introduced in Figure 2 to achieve $\mathbf{d}=\{1 / 3,1 / 12,1 / 24$, $1 / 192,1 / 1536\}$ number of DoFs for the transmitters. Total DoF number of 0.47 is achieved for this scheme which is still far less than the optimal DoF of 2 for this channel. Due to these difficulties, it is preferred if IA can be accomplished without or with limited symbol extensions. Actually, IA schemes are most likely to be used in MIMO networks. Feasibility of perfect IA is not yet available in theory for most MIMO networks. Iterative algorithms provide numerical insights into the feasibility of IA in these cases. In this section, distributed IA algorithm presented in [12] is extended to be utilized for the case of general IFC with multiple antenna nodes and with no symbol extensions.

Based on the system model presented in Section II, IA feasibility conditions are derived as follows,

$$
\begin{array}{r}
\mathbf{U}^{[j]{ }^{\dagger}} \mathbf{H}^{[j k]} \mathbf{V}^{[k]}=0, \forall k \notin S_{j} \\
\operatorname{rank}\left(\mathbf{U}^{[j]{ }^{\dagger}} \mathbf{H}^{[j \mathrm{k}]} \mathbf{V}^{[\mathrm{k}]}\right)=\mathrm{d}^{[\mathrm{k}]}, \forall \mathrm{k} \in \mathrm{S}_{\mathrm{j}}
\end{array}
$$

while the rank condition is typically assumed to be automatically satisfied, this is not true in the case of symbol extensions because channel matrices cannot be assumed to be generic. When no symbol extensions is applied and the MIMO channels $\mathbf{H}^{[j k]}$ are generic, the rank condition is satisfied with probability one. In other words, with generic channels there is no need to explicitly introduce the rank constraint into the optimization problem. Equation (23) requires that at each receiver, all interferences must be suppressed, leaving as many interference-free dimensions as the DoF allocated to that receiver.

The intention is to use alternating optimization procedure similar to [12], to find the transmit precoding and receive zeroforcing matrices. We start with arbitrary transmit and receive filters $V^{[k]}, U^{[j]}$ and iteratively update these filters to approach IA. Alternating optimization procedure is realized by switching the direction of communication. The total interference leakage at receiver $j$ due to all undesired transmitters is given by:

$I^{[j \star]}=\operatorname{Tr}\left[\mathbf{U}^{[\mathbf{j}]^{\dagger}} \mathbf{Q}^{[\mathbf{j}]} \mathbf{U}^{[\mathbf{j}]}\right]$ 
where

$\mathbf{Q}^{[j]}=\sum_{k=1, k \notin S_{j}} \frac{P^{[k]}}{d^{[k]}} \mathbf{H}^{[j k]} \mathbf{V}^{[k]} \mathbf{V}^{[k]^{\dagger}} \mathbf{H}^{[j k]^{\dagger}}$

Let us define $\overleftarrow{S}_{k}$ as the set of receivers that request message from transmitter $k$, i.e., $\overleftarrow{S}_{k}=\left\{j, j=[N], k \in S_{j}\right\}$ . In the reciprocal network, the total interference leakage at receiver $k$ due to all undesired transmitters $\overleftarrow{\bar{S}}=\left\{j, j=[N], k \notin S_{j}\right\}$ is given by (26):

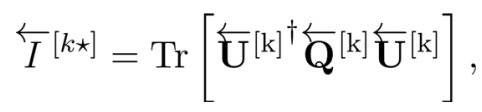

where

$$
\overleftarrow{\mathbf{Q}}^{[k]}=\sum_{j \in \overleftarrow{\bar{S}}_{k}} \frac{\overleftarrow{P}^{[j]}}{n^{[j]}} \overleftarrow{\mathbf{H}}^{[k j]} \overleftarrow{\mathbf{V}}^{[j]} \overleftarrow{\mathbf{V}}^{[j]^{\dagger}} \overleftarrow{\mathbf{H}}^{[k j]^{\dagger}}
$$

is the interference covariance matrix at receiver $k$. The iterative algorithm alternates between the original and reciprocal networks. In the original network, each receiver solves the following optimization problem.

$$
\min _{\mathbf{U}^{[j]}: M \times n^{[j]} \mathbf{U}^{[j]} \mathbf{U}^{[j]^{\dagger}}=\mathbf{I}_{n}[j]} I^{[j \star]}
$$

In other words, receiver $j$ chooses its interference suppression filter $\mathbf{U}^{[j]}$ to minimize the leakage interference due to all undesired transmitters. The $n^{[j]}$ dimensional received signal subspace that contains the least interference is the space spanned by the eigenvectors corresponding to the $n^{[j]}$ smallest eigenvalues of the interference covariance matrix $\mathbf{Q}^{[j]}$. Thus, the $n^{[j]}$ columns of $\mathbf{U}^{[j]}$ are given by:

$$
\mathbf{U}_{\star n}^{[j]}=\nu_{n}\left[\mathbf{Q}^{[j]}\right], n=1, \cdots, n^{[j]},
$$

where $\nu_{n}[\mathbf{A}]$ is the eigenvector corresponding to the $n^{\text {th }}$ smallest eigenvalue of $\mathbf{A}$.

For the second step, consider the reciprocal network obtained by reversing the roles of the transmitters and the receivers. The transmit precoding matrices in the reciprocal network, $\overleftarrow{\mathbf{V}}[j]$, are the receive interference suppression matrices $\mathbf{U}^{[j]}$ from the original network. Each receiver in the reciprocal network solves the following optimization problem.

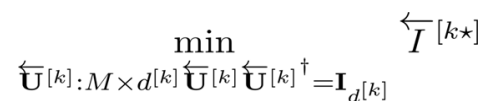

Similarly, the $d^{[k]}$ columns of $\overleftarrow{\mathbf{U}}^{[k]}$ are given by:

$$
\overleftarrow{\mathbf{U}}_{\star d .}^{[k]}=\nu_{d}\left[\overleftarrow{\mathbf{Q}}^{[k]}\right], d=1, \cdots, d^{[k]}
$$

The receive zero-forcing filters in the reciprocal network are then used as the transmit precoding matrices in the original network, and the algorithm iterates again. The iterations continue in this manner until the algorithm converges. The proof of convergence for this algorithm is similar to the one presented in [12]. It can be observed that each step in the algorithm reduces the value of the total leakage interference. The total leakage interference for general IFC is defines as follows,

$$
I_{w}=\sum_{j=1}^{N} I^{[j \star]}
$$

where $I^{[j \star]}$ is defined in (24).

The algorithm presented in this section seeks perfect IA. In particular it seeks to create an interference free subspace of the required number of dimensions that is designated as the desired signal subspace. However, note that perfect IA may not always be feasible. In such scenarios, the network structure should be reconfigured. Simulation results indicate that residual interference due to the imperfect IA significantly degrades the achievable sum rate performance in high signal to noise ratios. We can either reduce the number of transmitted streams, increase the number of receive antennas, use relays in the network to accommodate IA, or use channel extension to make IA feasible. In the following, we will consider the case of reduced number of transmitted streams (RNS) and increased number of receive antennas (INA) along with the case of optimal DoF assignment (ODA).

\section{Simulation PESUlts}

In this section we run simulations to illustrate practical feasibility of the optimal DoF assignment. Consider the $6 \times 3$ user regular IFC shown in Figure 2. It is proved that perfect IA is not feasible for this network, assuming single antenna nodes. We consider a MIMO interference channel where each transmitter/receiver is equipped with 3 antennas. Optimal DoF assignments for this structure is obtained as $d^{[k]}=1, k=[6]$. The system of bilinear equations for IA requirements of this network constitutes an improper system because the number of the independent variables is 18 which is less than the number of equations which is 24 . Therefore, we expect the perfect IA to be still infeasible. This fact is confirmed with simulation results.

We allocate power $1 / d$ to each column of the precoding matrices, and set the noise power level to $\sigma^{2}=10^{-P 11}$, where $P=[0: 10: 60] d B$. Final results are obtained by averaging over 200 channel realizations, where each channel element is i.i.d. zero mean unit variance circularly symmetric complex Gaussian. We run $10^{4}$ iterations of the extended minimum leakage algorithm for each simulation. We plot the sum rate of each system. The sum rate is computed as $R=\sum_{j=1}^{N} \log \operatorname{det}\left(\mathbf{I}_{d^{[j]}}+\left(\sigma^{2} \mathbf{I}_{d^{[j]}}+\mathbf{Q}^{[j]} \mathbf{Q}^{[j]^{\dagger}}\right)^{-1} \mathbf{S}^{[j]} \mathbf{S}^{[j]}{ }^{\dagger}\right)$, where $\mathbf{S}^{[j]}$ is defined as the covariance matrix of desired signal for receiver $j$. It is observed in Figure 3 that the sum rate of ODA case is limited with residual interference at high signal to noise ratios. Sum rate performance is also plotted for two cases of RNS ( $\left.d^{[1]}=0, d^{[k]}=1, k=2\right)$ and INA (4 receive antennas). We compare the performance of the iterative IA algorithms for this channel with orthogonal schemes and isotropic transmission. 
The sum rate for the orthogonal scheme is calculated assuming equal time sharing for the users, and with power $6 P$ per node. The isotropic transmission case refers to the case where each transmitter sends $d^{[k]}=1, k=[6]$ stream of equal power regardless of the channel information.

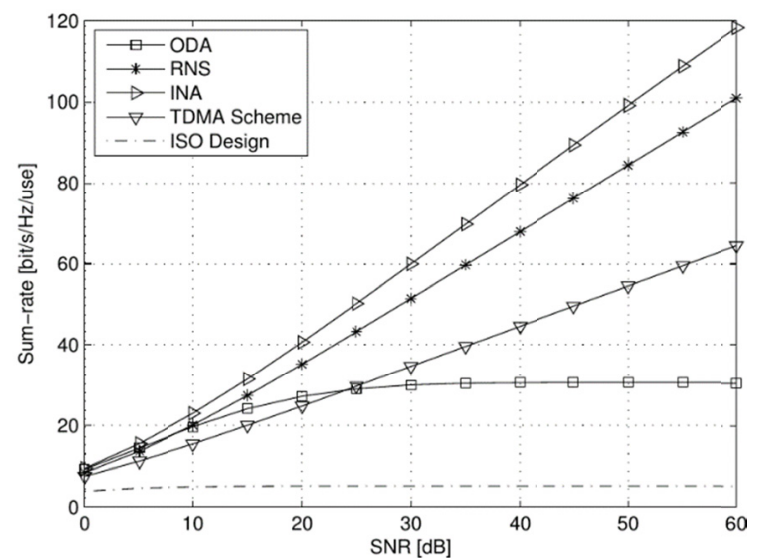

Fig. 3. Performance of the iterative IA algorithm for the $6 \times 3$ user regular IFC.

It is observed that orthogonal schemes outperform ODA at high signal to noise ratios in this specific structure. Although the upperbound on the DoF for this network with 3 antennas at each receiver node is 6 , approaching the upperbound may entail channel extensions. The slope of the INA sum rate at high signal to noise ratios suggests that 6 degrees of freedom $\left(d^{[1]}=\ldots=d^{[6]}=1\right)$ can be achieved by using 4 antennas at the receiver nodes without channel extension. For feasible IA, leakage interference for each of three receivers should be zero. It is realized that IA is feasible in case of RNS and INA. Note that, the system of the bilinear equations is proper for both of these cases $N_{v}=N_{e}=16$ for RNS scheme and $N_{v}=N_{e}=24$ for INA scheme.

Iterative algorithm can also be used to check theoretical feasibility of IA strategy for a given number of streams per user. We evaluate the iterative algorithm for a $5 \times 3$ IFC with requested message sets defined as $S_{1}=\{1,5\}, S_{2}=\{1,2\}$ and $S_{3}=\{3,4,5\}$. Solving the linear programming (6), optimal DoF assignment for this network is obtained as $d^{[1]}=d^{[2]}=0.4$, $d^{[3]}=d^{[4]}=d^{[5]}=0.2$. We have assumed 5 antennas at each receiver node for the simulations. The system of bilinear equations is proper in this case, $N_{v}=40, N_{e}=36$, hence, ODA is likely to be feasible for this scenario. The average number of received DoFs is $\sum_{j=1}^{3} n^{j} / N=10 / 3$. This measure provides us with an idea of whether the algorithms converge to a perfect IA solution or not. Hence, if perfect alignment is achieved, i.e., the interference is perfectly suppressed and the direct channels are full-rank, the slope of the sum rate at high signal to noise ratios should be close to $10 / 3$. Figure 4 suggests that perfect IA is feasible. The slope of the sum rate is approximately 3 at high signal to noise ratios. Evaluating leakage interference in desired signal space confirms the fact that perfect IA is feasible in this scenario and there is no need for the RNS and INA strategies in this network. In fact, RNS has less sum rate than ODA in this case, which emphasizes the benefits of using optimal DoF assignment.

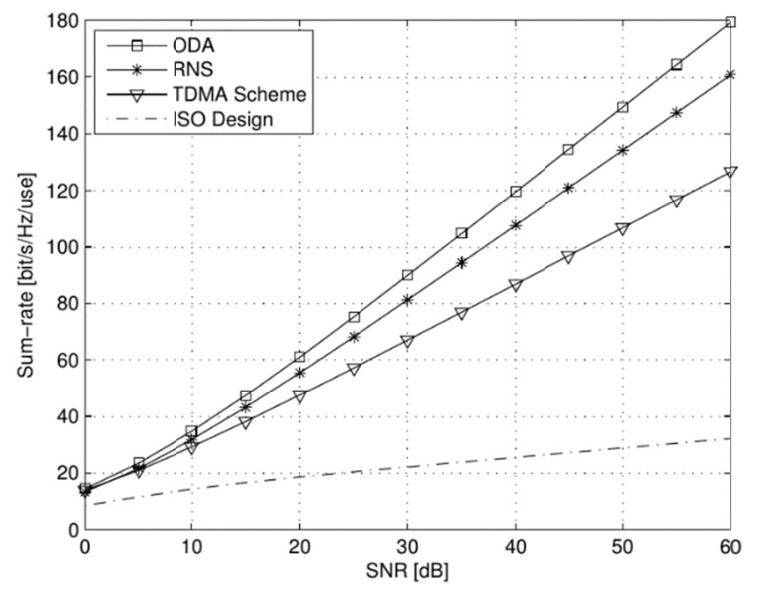

Fig. 4. Performance of the iterative IA algorithm for the $5 \times 3$ general IFC.

\section{CONCLUSION}

The concept of regular interference network is introduced as an interference network in which all active transmitters have equal number of optimal DoF. Regular IFCs are mostly encountered in networks with few number of users. It is proved that perfect IA cannot be achieved for the single antenna regular IFCs with generic channel coefficients. The feasibility of the optimal DoF assignment for the general interference networks is not easily mathematically tractable. Distributed minimum leakage IA is extended to work with general IFC. This algorithm is based on the network reciprocity. Simulation results suggest that, despite the non-symmetric nature of the general IFC, iterative IA algorithm is still useful in practical realization of IA and evaluation of the perfect IA feasibility for this network. Numerical comparisons to orthogonal schemes and simultaneous isotropic transmission schemes show that the benefits of using distributed IA algorithms are significant.

\section{REFERENCES}

[1] R. H. Etkin, D. N. C. Tse, H. Wang, "Gaussian interference channel capacity to within one bit", IEEE Transactions on Information Theory, Vol. 54, No. 12, pp. 5534-5562, 2008

[2] S. Jafar, M. Fakhereddin, "Degrees of freedom for the MIMO interference channel,", IEEE Transactions on Information Theory, Vol. 53, No. 7, pp. 2637-2642, 2007

[3] A. Host-Madsen, A. Nosratinia, "The multiplexing gain of wireless networks", IEEE International Symposium on Information Theory, Adelaide, Australia, pp. 2065-2069, September 4-9, 2005

[4] M. A. Maddah-Ali, A. S. Motahari, A. K. Khandani, "Communication over MIMO X channels: Interference Alignment, Decomposition, and Performance Analysis", IEEE Transactions on Information Theory, Vol. 54, No. 8, pp. 3457-3470, 2008

[5] S. A. Jafar, S. Shamai, "Degrees of freedom region for the MIMO X channel", IEEE Transactions on Information Theory, Vol. 54, No. 1, pp. $151-170,2008$

[6] V. R. Cadambe, S. A. Jafar, "Interference alignment and degrees of freedom of the $\mathrm{K}$-user interference channel", IEEE Transactions on Information Theory,Vol. 54, No. 8, pp. 3425-3441, 2007 
[7] C. M. Yetis, T. Gou, S. Jafar, A. H. Kayran, "On feasibility of interference alignment in MIMO interference networks", IEEE Transactions on Signal Processing, Vol. 58, No. 9, pp. 4771-4782, 2010

[8] M. Razaviyayn, G. Lyubeznik, L. Zhi-Quan, "On the degrees of freedom achievable through interference alignment in a MIMO interference channel", IEEE Transactions on Signal Processing, Vol. 60, No. 2, pp. $812-821,2011$

[9] T. Liu, C. Yang, "On the feasibility of linear interference alignment for MIMO interference broadcast channels with constant coefficients", IEEE Transactions on Signal Processing, Vol. 60, No. 9, pp. 2178-2191, 2013

[10] L. Ruan, V. N. Lau, M. Z. Win, "The feasibility conditions for interference alignment in MIMO networks", IEEE Transactions on Signal Processing, Vol. 61, No. 8, pp. 2066-2077, 2013

[11] L. Ke, A. Ramamoorthy, Z. Wang, H. Yin, "Degrees of freedom region for an interference network with general message demands", IEEE Transactions on Information Theory, Vol. 58, No. 6, pp. 3787-3797, 2012

[12] K. Gomadam, V. Cadambe, S. Jafar, "A distributed numerical approach to interference alignment and applications to wireless interference networks", IEEE Transactions on Information Theory, Vol. 57, No. 6, pp. 3309-3322, 2011

[13] Z. Samadi, V. T. Vakili, F. Haddadi, "Channel aided interference alignment”, IET Signal Processing, Vol. 11, No. 7, pp. 854-860, 2017

\section{AUTHORS PROFILE}

Zainalabedin Samadi was born in Hashtroud, Iran, on April 26, 1985. He received the B.S. degree from the Ferdowsi University of Mashhad, Mashhad, Iran, in 2008, the M.S. degree from Iran University of Science and Technology, Tehran, Iran, in 2011. He is currently working toward the Ph.D. in the Department of Electrical Engineering, Iran University of Science and Technology. His research interests include multiuser information theory, joint source-channel coding, spread-spectrum systems and emerging interference alignment techniques.

Vahid Tabataba Vakili received the B.S. degree from Sharif University of Technology, Tehran, Iran, in 1970, the M.S. degree from the University of Manchester, Manchester, U.K., in 1973, and Ph.D. from the University of Bradford, Bradford, U.K., in 1977, all in electrical engineering. In 1985, he joined the Department of Electrical Engineering, Iran University of Science and Technology, Tehran. In 1997, he was promoted to Associate Professor. He has served as the Head of the Communications Engineering Department and as the Head of postgraduate studies. His research interests are in the areas of mobile cellular systems, interference cancellation for CDMA systems, and space-time processing and coding.

Farzan Haddadi was born in 1979. He received B.Sc., M.Sc., and Ph.D. degrees in communication systems from Sharif University of Technology, Tehran, Iran, in 2001, 2003, and 2010, respectively. In 2011, he joined Iran University of Science and Technology Faculty, Tehran, Iran. His research interests include array signal processing, statistical signal processing, nondestructive testing, sonar systems, and detection theory. 